« Le néoplatonisme, la poésie et la musique de la nature : Cudworth, Newton, Coleridge, Emerson ", Circulation des savoirs et reconfiguration des idées. Perspectives croisées France Brésil, Laurence Dahan-Gaida (dir.), Villeneuve-d'Ascq, Presses Universitaires du Septentrion, 2016.

\title{
Le néoplatonisme, la poésie et la musique de la nature : Cudworth, Newton, Coleridge, Emerson
}

Danielle Follett

Université de Franche-Comté

Les idées ne sont pas des archétypes ou des essences platoniciennes qui existent en dehors de l'histoire, mais elles ont des origines qui peuvent être retracées; elles connaissent des développements, des croisements, des déclins, des réapparitions. Elles sont inséparables des différents contextes culturels et historiques dont elles sont les expressions plus ou moins stables et dont elles portent l'empreinte. Hétérogènes, dynamiques et complexes, les idées sont constituées par des assemblages de notions et de mots reliés entre eux selon des modalités diverses. Les idées ne sont pas équivalentes aux mots, qui ont leur propre histoire et leur propre temporalité. Une idée est plutôt constituée d'une constellation de termes et d'usages qui sont liés par des relations d'affinité plus ou moins explicites et sont variables historiquement et culturellement. Un exemple de ces variations serait l'ensemble épistémique formé par les termes peuple / population / communauté / populace / tribu / multitude / masses / foule / démos ; un autre exemple de telles constellations conceptuelles serait l'ensemble hasard / contingence / aléa / indétermination / chance / fortune. La non-identité entre mot et idée ne signifie pas que les idées soient des essences supra-historiques se manifestant à travers des mots : elles sont bien plutôt des constructions conceptuelles contingentes et complexes, des produits de l'histoire réelle. Elles varient et évoluent dans le temps, comme en témoignent par exemple les mutations, très riches, de l'idée de libéralisme. Selon les périodes historiques, elles peuvent décliner, disparaître ou renaître.

L'objet de cet article sera, à partir d'exemples précis, d'étudier l'histoire de l'idée de la musique de la nature, en la replaçant dans une perspective à la fois diachronique et comparatiste. 
Il s'agira de mettre en évidence ses implications épistémologiques et idéologiques, ainsi que le jeu des imbrications, croisements, greffes, qu'elle a connu, entre différents domaines des savoirs (musique, philosophie, religion, sciences, psychologie, littérature), périodes (du XVIIe au XIXe siècle) et pays (Angleterre, États-Unis). D’autres pays, auteurs et périodes pourraient figurer dans une étude sur la musique de la nature ; faute de place, on se focalisera ici sur Cudworth, Newton, Coleridge et Emerson, avec nécessairement une perspective synthétique.

Le tintement simple d'un instrument éolien faisant entendre, au gré des bourrasques et des accalmies, des cliquetis lents et entrecoupés, des fracas en cascade, parfois le silence, semble avoir peu de rapport avec la métaphysique. Cependant, la harpe éolienne devient une figure de la musique de la nature, idée qui comporte des dimensions métaphysiques et poétiques. La harpe éolienne ressemble à une cithare, placée soit dans l'encadrement d'une fenêtre soit en plein air dans la nature. Elle se compose d'une caisse de résonnance en bois et de cordes au travers desquelles le vent s'engouffre, produisant ainsi des sons. Cet objet élémentaire devient une figure poétique, d'abord au XVIII ${ }^{\mathrm{e}}$ siècle en Angleterre, puis à l'époque romantique dans les autres pays européens et aux États-Unis. Cette particularité de la harpe éolienne à rendre sonores les forces naturelles fait que sa musique sera considérée comme celle de la nature elle-même, sa « voix » comme la voix de la nature.

L'idée de la «musique des sphères", traditionnellement attribuée à Pythagore, est relayée par Platon, Cicéron et de nombreux autres penseurs antiques. Il s'agit d'une théorie de l'harmonie universelle inaudible qui ordonnerait la nature, le monde et l'univers. Cette philosophie néoplatonicienne pousse Johannes Kepler à rechercher les lois régissant un univers régulier et « harmonique », ainsi qu'en témoigne son Harmonices Mundi de 1619 dans lequel il décrit les découvertes astronomiques qui fondent notre compréhension du système solaire ${ }^{1}$. Un souci semblable, comme nous le verrons, pousse à son tour Isaac Newton à étudier l'astronomie ainsi que le phénomène de la gravitation. Le néoplatonisme est une doctrine philosophique et religieuse développée par des penseurs dans la tradition platonicienne, surtout Plotin, Proclus, Jamblique et Porphyre au $\mathrm{III}^{\mathrm{e}}$ siècle. Il s'agit d'une forme d'immanentisme dynamique qui postule différentes strates de l'existence, toutes reliées et créées par l'Un (le Noûs), la source divine. Selon cette philosophie, l'énergie divine en provenance de l'Un circule à travers ces

\footnotetext{
${ }^{1}$ Sur la musique des sphères, voir Otto Kinkeldey, "The Music of the Spheres », Bulletin of the American Musicological Society, n ${ }^{\mathrm{o}}$ 11-12-13, 1948, p. 30-32.
} 
strates concentriques; la force qui émane de la source unique crée et anime à la fois l'existence entière. L'énergie divine trouve son analogue dans le vent qui traverse la harpe, « harmonisant » l'existence. Cette philosophie se distingue du platonisme dualiste strict en ceci que sa métaphysique d'immanence tend vers un monisme panthéiste, mais elle comporte toutefois un aspect dualiste dans la mesure où elle voit une différence qualitative et non seulement de degré entre la source de l'énergie divine et ses créations dans l'univers.

Les «platoniciens de Cambridge » de la deuxième moitié du XVII ${ }^{\mathrm{e}}$ siècle, Ralph Cudworth et Henry More, s'inscrivent dans la tradition néoplatonicienne. Ils adoptent une philosophie qui est orientée contre, d'une part, les «athées hobbesiens » et, de l'autre, les hérétiques panthéistes (spinozistes ou hylozoïstes, qui pensent que la matière est vivante et animée). Leur perspective se distingue aussi de la philosophie de la nature entièrement mécanisée de Descartes, car celle-ci n'est pas complètement étrangère à l'athéisme en ceci que le créateur est absent de la nature. Ces penseurs créent donc une association quelque peu instable de dualisme et d'immanentisme. La matière doit être inerte et passive pour éviter l'hérésie panthéiste, mais il faut aussi qu'il y ait une force spirituelle immanente dans la nature, au moins à un certain degré, pour éviter le danger du mécanisme cartésien. Selon la tradition néoplatonicienne classique, la nature est une émanation divine éloignée de sa source - éloignée, mais toujours divine.

Cudworth, dans son influent ouvrage intitulé Le Système véritable intellectuel de l'univers (1678), donne comme nom à la force immanente «la nature plastique » («Plastic nature »), un concept qui joue un rôle semblable à celui de l'anima mundi néoplatonicienne (ou, pour anticiper, comparable au terme emersonien de «Sur-âme » ou d' « Oversoul »), laquelle est une force médiatrice entre la source divine et le monde, l'animant avec ses émanations nouménales. Cudworth écrit, dans le contexte d'une description de la nature plastique, le passage suivant :

Les mythes anciens représentaient la nature de l'univers comme Pan jouant sur une pipe ou une harpe, et amoureux de la nymphe Echo ; comme si la nature, à travers une sorte de 
mélodie silencieuse, faisait partout danser en bonne harmonie et proportion toutes les parties de l'univers...

Plus loin, Cudworth décrit Pan :

PAN... était censé ... jouer sur le monde comme sur un instrument musical... [et] par ce mot les Arcadiens et les Grecs voulaient dire, non pas le monde corporel inanimé, ni un monde doté d'une nature insensée uniquement, mais un monde qui procède d'un principe intellectuel ou un esprit divin, qui l'encadre harmonieusement; et qui est toujours maintenu accordé, et dirigé et gouverné par cet esprit. On dit que cet esprit est le pasteur universel de toute l'humanité et du monde.... Pan est donc le seul, unique Dieu (parce qu'il ne peut pas y avoir plus qu'un seul Pan, plus qu'un tout ou un univers) qui contient tout à l'intérieur de lui-même, qui montre tout à partir de lui-même, qui encadre le monde harmonieusement, et qui est d'une certaine manière tout ce qui existe. ${ }^{3}$

On a ici à la fois un dualisme, entre musicien et monde (l'esprit et l'instrument), et un immanentisme quasi-moniste (Pan est tout ce qui existe). Le statut de Pan est quelque peu ambigu, car il est à la fois le joueur de la flute et la flute, ou l'esprit divin et la nature qui est l'instrument traversé par l'esprit divin. Ailleurs, Cudworth dit que Pan est Dieu, le « gouverneur » de l'univers :

Pan, ou «l'univers », était compris fréquemment par les théologiens païens aussi... comme le Dieu, avec toute sa fécondité, Dieu qui se manifeste dans le monde ; ou bien, comme Dieu et le monde ensemble à la fois, ce dernier ayant été considéré comme uniquement une émanation ou un efflux à partir du premier. [...] Le Pan arcadien n'était pas le monde corporel seulement, mais surtout le gouverneur du monde... le Dieu qui se manifeste dans le monde, et omniprésent dans tout ce qui existe. ${ }^{4}$

\footnotetext{
${ }^{2}$ Ralph Cudworth, The True Intellectual System of the Universe, 3 vol., Bristol, Thoemmes, 1995, vol. 1, p. 242. Toutes les traductions des citations sont de l'auteur de cet article.

${ }^{3}$ Ibid., vol. 2, p. 208-209.

${ }^{4}$ Ibid., vol. 1, p. 582-583.
} 
L'harmonie du monde est le résultat du principe actif qui se diffuse à travers lui, comme le vent de Pan à travers sa flute :

Les anciens pensaient que Pan, c'est-dire, la nature, jouait sur une harpe... Le cerveau d'un être rationnel et intellectuel sera ravi et transporté avec enthousiasme quand il le contemple, et dansera de sa propre volonté à la musique de cette pipe de Pan, la musique et l'harmonie intellectuelles de la nature. ${ }^{5}$

Pour Cudworth, l'identité de Pan, principe actif dans la nature, ne se sépare pas entièrement de la nature. Cette instabilité entre dualisme et monisme immanentiste marquera profondément la métaphysique de la harpe éolienne.

Dans ses recherches sur les précurseurs de sa propre pensée, Isaac Newton, contemporain et ami des platoniciens de Cambridge, porte un grand intérêt à la musique des sphères comme expression de l'harmonie divine ${ }^{6}$. Selon lui, Pythagore avait déjà formulé les éléments mathématiques de la gravitation et de la relation des sept planètes au soleil, les exprimant comme les harmoniques des sept cordes de la lyre d'Apollon. Newton s'interroge sur la «cause » de la force universelle de la gravitation qui agit sur la matière :

Par quel moyen agissent les corps l'un sur l'autre à une distance. Et à quel Agent attribuaient les Anciens la gravitation de leurs atomes et qu'est-ce qu'ils voulaient dire quand ils appelaient Dieu une harmonie et comparaient Dieu et la matière (la partie corporel de l'Univers) au Dieu Pan et sa Pipe. ${ }^{7}$

La matière, selon Newton, est passive ; mais il existe une force immanente qui l'anime, que les anciens appelaient «l'harmonie » et qu'il appelle la gravitation, la «vie» («life») ou Dieu. Cette force divine entretient la même relation avec la matière que Pan avec sa flute. Dans un autre passage, Newton écrit :

\footnotetext{
${ }_{6}^{5}$ Ibid., vol. 3, p. 600 .

${ }^{6}$ Voir J. E. McGuire et P. M. Rattansi, «Newton and the "Pipes of Pan" », Notes and Records of the Royal Society of London, vol. 21, $\mathrm{n}^{\mathrm{o}} 2$ 2, 1966, p. 108-143, qui traite des manuscrits théologiques inédits de Newton ; voir aussi Danton B. Sailor, « Newton's Debt to Cudworth », Journal of the History of Ideas, vol. 49, nº 3, July-Sept. 1988, p. 511-518.

${ }^{7}$ Manuscrit dans la bibliothèque universitaire de Cambridge, Ad. MS 3970.f.292v, cité dans J. E. McGuire et P. M. Rattansi, «Newton and the "Pipes of Pan"», op. cit., p. 108.
} 
Et comme... toutes les choses ont été créées avec un art et une sagesse parfaits et la nature ne fait rien inutilement, si la vie universelle et tout l'espace existent à partir du sensorium d'un être pensant qui par sa présence immédiate y perçoit toutes les choses, alors ces lois du mouvement qui proviennent de la vie pourraient être d'une étendue universelle. Les Philosophes anciens semblent avoir évoqué de telles lois quand ils appelaient Dieu l'Harmonie, et quand ils représentaient sa façon d'activer la matière harmonieusement avec le Dieu Pan qui joue sur une Pipe, et pensaient, en attribuant une musique aux sphères, que les distances et les mouvements des corps célestes étaient harmonieux, et représentaient les Planètes avec les sept cordes de la Harpe d'Apollon. ${ }^{8}$

Comme dans la tradition néoplatonicienne, Newton établit une analogie entre la vie universelle et intelligente qui agit à l'intérieur de la matière et l'esprit humain individuel qui meut son corps de l'intérieur. Cette «vie » est la source des lois universelles de la nature et donc de l'harmonie, à laquelle les anciens avaient associé la figure de Pan, le dieu qui crée de la musique en jouant avec la nature. La musique des sphères est le résultat du contact de la force divine avec la matière inerte, son instrument; cette force «active la matière de façon harmonieuse ». L'intervention divine constante dans la création est l'un des principes newtoniens, car sans apport nouveau d'énergie divine la matière se désintègre. Cette idée fut ridiculisée par Leibniz qui, dans une critique restée célèbre, dit qu'un Créateur parfait ne devrait pas avoir besoin d'entretenir régulièrement sa création; la réponse des newtoniens est qu'il est facile d'imaginer que, sans présence divine immanente (au moins intermittente), l'univers soit éternel et qu'il n'y ait jamais eu d'acte de création - et donc qu'il n'y ait pas de créateur. Newton théorise cette force immanente comme un ensemble de «principes actifs»: "Puisque la variété du Mouvement que l'on trouve dans le monde diminue constamment, il y a une nécessité de le conserver et de l'alimenter avec des Principes actifs $»{ }^{9}$.

Newton hésite sur la question de savoir si ces principes actifs sont matériels, comme l'éther ou - sa dernière hypothèse - comme les comètes qui font circuler l'énergie divine à travers le cosmos et ravitaillent le soleil, ou s'ils sont seulement immatériels, comme la

\footnotetext{
${ }^{8}$ Manuscrit d'une version de Opticks, ibid., p. 118

${ }^{9}$ Newton, Opticks, New York, Dover, 1952, p. 399. Voir David Kubrin, « Newton and the Cyclical Cosmos: Providence and the Mechanical Philosophy », Journal of the History of Ideas, vol. 28, n 3, 1967, p. 331-332.
} 
« volonté » divine. Si dans sa philosophie, ces deux hypothèses reviennent finalement au même (car la cause immatérielle prend une forme matérielle), il est certain que Newton est très soucieux d'apporter une réponse scientifique à la manifestation matérielle du principe actif ${ }^{10}$. Les forces divines actives et immanentes, comme celle que Newton rend responsable de la gravitation, jouent le rôle d'agent qui anime et « harmonise » les corps passifs - comme Pan avec sa flûte et comme le vent qui traverse la harpe ${ }^{11}$. Les principes actifs de Newton sont donc apparentés aux forces divines immanentes décrites par Cudworth, More et les autres platoniciens de Cambridge ${ }^{12}$.

Ces idées néoplatoniciennes auront une grande influence sur le jeune Coleridge. Jusqu'aux alentours de 1800, alors que Coleridge a vingt-huit ans, il maintient avec véhémence une perspective religieuse et philosophique quasi-panthéiste. Déjà lycéen, il s'était immergé dans la tradition néoplatonicienne de Plotin, de Proclus et des platoniciens de Cambridge, ainsi que dans le mysticisme chrétien de Jakob Boehme. Le jeune Coleridge compose un mélange particulier et assez cohérent entre le néoplatonisme qu'il avait découvert tôt, l'unitarisme chrétien qu'il adopte quand il va étudier à l'université de Cambridge, et l'associationnisme, c'est-à-dire la psychologie empiriste de David Hartley. Hartley (1705-1757), dont Coleridge donnera le patronyme à son fils en second prénom, David Hartley Coleridge (né en 1796), est le fondateur de l'école psychologique et physiologiste associationiste.

Avant de pouvoir expliquer la philosophie du jeune Coleridge et son mélange de néoplatonisme et d'empirisme hartleyen, il faudrait aborder un sujet sur lequel ces deux philosophies sont en franc désaccord: l'épistémologie ou la philosophie de la connaissance humaine. Si Newton est très influencé par la pensée néoplatonicienne de Cambridge sur les sujets ontologiques et théologiques, il ne l'est pas sur les questions épistémologiques qui seront brûlantes vers 1690 - juste après la mort de More et de Cudworth en 1687 et 1688 - avec la

\footnotetext{
${ }^{10}$ Pour une discussion de l'histoire de l'idée de la force en physique d'Aristote à Einstein en passant par Newton, et en particulier la nature de la gravitation, voir Alexis de Saint-Ours, «Des Forces aux Champs : L'exemple de la gravitation », in TLE (Théorie Littérature Enseignement), $\mathrm{n}^{\mathrm{o}} 24$ : «Forces-figures. Faire sentir les forces insensibles », Presses Universitaires de Vincennes, 2006-2007, p. 23-38.

${ }^{11}$ J. E. McGuire et P. M. Rattansi décrivent les recherches de Newton sur la musique des sphères et le principe de gravitation universelle comme une tentative de comprendre la nature de la force divine, « Newton and the "Pipes of Pan"», op. cit., p. 138.

${ }^{12} \mathrm{C}$ 'est donc une erreur de penser que le système newtonien est sans dynamisme ; ses lois mécaniques ne décrivent que la partie passive et corporelle de l'univers, et non la force divine qui l'anime. Voir Kubrin, « Newton and the Cyclical Cosmos », op. cit., p. 330. Kubrin montre que pour Newton, la désintégration progressive de la nature fait que son dynamisme est probablement cyclique, avec la destruction et la création des mondes qui sont en eux-mêmes relativement stables.
} 
publication de l'Essai sur l'entendement humain de Locke en 1689. Les néoplatoniciens constituent la cible principale de cet ouvrage qui nie l'existence des idées innées et pose les fondements épistémologiques de la recherche scientifique empiriste. En matière de philosophie de la connaissance, Newton suit clairement Locke et le cercle de penseurs anti-platoniciens de la Royal Society ${ }^{13}$. Selon les néoplatoniciens, le platonisme et sa doctrine des idées innées n'est en rien incompatible avec la science, ni même avec le mécanisme; Bacon, père fondateur de l'empirisme, n'est pas étranger au platonisme. Pour eux, la science est la recherche des lois éternelles et divines reflétées dans la nature, qui sont aussi présentes dans l'esprit de l'homme. Or, pour Locke et les praticiens de la nouvelle science, cette idée engendre des erreurs scientifiques, car elle justifie la méthode déductive et l'abus des hypothèses. Le cerveau de l'homme est donc une tabula rasa, sans contenu à la naissance et tout ce qui s'y trouve y est entré par la voie de l'expérience sensorielle. Il est intéressant de noter que sur les questions ontologiques et théologiques, plus précisément sur la question des causes première et finale, Locke est moins tranché que lorsqu'il s'agit de questions épistémologiques. Son attitude restera quelque peu agnostique sur le sujet de recherche auquel Newton consacre sa vie : une explication de la cause de la gravitation. Locke rend l'esprit humain aussi passif que la matière et relègue la question ontologique de la «cause» première du mouvement des corps aux provinces théologiques, au-delà de notre compréhension humaine. Ainsi, il défait l'analogie platonicienne entre l'esprit individuel actif et l'Esprit nouménal, préparant ainsi la voie au nécessitarisme du XVIII $^{\mathrm{e}}$ siècle.

L'associationnisme de Hartley, qui s'inscrit dans la lignée empiriste de Locke et s'inspire aussi du mécanisme physique de Newton, considère le cerveau humain comme une machine sensible qui reçoit des impressions sensorielles du monde extérieur et agit en fonction de ces influences. Hartley développe les « doctrines de la vibration et de l'association », le sujet de la première partie de son ouvrage, Observations sur l'homme, son cadre, ses devoirs et ses attentes (1749). Des sensations découlent tous les autres phénomènes psychologiques : l'émotion, la raison, la mémoire, les pensées et même les actions sont expliquées par les lois de l'association. La sensation est le résultat des vibrations subtiles des nerfs ultra-sensibles; la mémoire et les

\footnotetext{
${ }^{13}$ Voir deux articles de G. A. J. Rogers, «Locke's Essay and Newton's Principia », Journal of the History of Ideas, vol. 39, $\mathrm{n}^{\circ}$ 2, Apr.-Jun., 1978, p. 217-232 ; «Locke, Newton, and the Cambridge Platonists on Innate Ideas », Journal of the History of Ideas, vol. 40, $\mathrm{n}^{\circ}$ 2, Apr.-Jun., 1979, p. 191-205 ; Rogers avance l'idée que c'est Newton qui est influencé par Locke et non l'inverse, comme on le pense souvent. Voir aussi Lisa J. Downing, «Locke's Newtonianism and Lockean Newtonianism », Perspectives on Science, 1997, vol. 5, nº 3, p. 285-310.
} 
pensées relèvent du fait que le cerveau continue à vibrer même sans stimulus externe, et ces vibrations dans le cerveau forment des associations ou des liens machinaux. Hartley est considéré comme l'un des fondateurs de l'école de psychologie qui essaie d'établir des liens intimes entre les phénomènes psychiques et physiologiques, et il influencera John Stuart Mill.

Le jeune Coleridge se considère « nécessitarien » en référence à ces idées. Hartley, ainsi que l'unitarien Joseph Priestley comme de nombreux autres penseurs déistes et rationalistes à cette époque, épouse une métaphysique déterministe qui reste pourtant chrétienne. La nature est considérée comme un système uni et harmonieux dont le fonctionnement est déterminé par les lois divines, comme celle de la causalité, et l'être humain participe à ce système comme toute autre entité naturelle. Pour Hartley, qui avait intitulé l'une des sections de son ouvrage «Remarques sur le mécanisme de l'esprit humain », l'être humain, comme tous les êtres et phénomènes, est une sorte de machine qui fait partie de la grande machine de la nature créée et mise en mouvement par Dieu. Coleridge peut donc écrire dans une lettre de 1794 : « Je suis un Chrétien Unitarien et un partisan de l'automatisme de l'homme $»^{14}$.

Dans la philosophie de jeunesse de Coleridge, l'association du dynamisme néoplatonicien de Cudworth et de l'empirisme lockéen de Hartley est donc tirée de deux traditions philosophiques concurrentes, mais ce mélange instable n'est pas entièrement contradictoire. Coleridge combine l'immanentisme ontologique des néoplatoniciens avec l'épistémologie hartleyenne du cerveau passif. Mais la théologie naturelle et l'unitarisme fondamental - Newton et Locke sont aussi proches des idées unitariennes - sont partagés par les deux courants. L'agnosticisme de Locke sur la nature de Dieu et des causes premières n'offre rien qui contredise l'ontologie néoplatonicienne. Ce mélange particulier entre le monisme néoplatonicien, l'unitarisme, le matérialisme et l'empirisme des lumières, sans oublier la «littérature de la sensibilité » de cette période ${ }^{15}$, crée un contexte fécond pour les premiers poèmes de Coleridge. Ces poèmes sont parsemés de références à un Dieu unique et omniprésent, auteur de toutes les actions. L'esprit humain est comme un instrument passif, traversé par l'énergie divine. Par exemple, on trouve une expression de cette conviction dans «Le Destin des nations » (1796) :

\footnotetext{
${ }^{14}$ «I am a Unitarian Christian and an Advocate for the Automatism of Man », Collected Letters of Samuel Taylor Coleridge, Earl Leslie Griggs (éd.), 6 vol., Oxford : Clarendon, 1956-1971, vol. 1, p. 147.

${ }^{15}$ Ce terme désigne généralement la littérature anglaise de la dernière moitié du XVIII ${ }^{\text {e }}$ siècle. Voir Northrop Frye, « Towards Defining an Age of Sensibility », ELH, vol. 23, n 2, 1956, p. 144-152.
} 
«L'Energie vaste et toujours agissante! " ${ }^{16}$. Remarquons le vocabulaire dynamique, néoplatonicien, de ce passage. Dans «Sur l'observation d'une fleur, le $1^{\text {er }}$ février 1796 », le poète s'adresse à une fleur qui s'épanouit trop tôt, pendant une période anormale de chaleur hivernale :

Et les séductions chaleureuses de ce jour ensoleillé

Tremblent sur mon corps, et harmonisent

Le cerveau tempéré, pour que même les pensées les plus tristes

Mélangent avec des sensations douces, comme des mélodies dures

Jouées habilement sur un instrument aux tonalités lisses.

$(\text { vers } 22-26)^{17}$

Les sensations et les séductions du jour ensoleillé d'hiver produisent un effet d'harmonisation avec le cerveau «tempéré » du poète, qui y répond comme un instrument de musique. Ce passage aux allures hartleyennes montre comment la figure du cerveau, en tant qu'instrument de musique, se prête à l'associationnisme empiriste. Les nerfs qui vibrent sont comme les cordes sensibles de l'instrument, et les sensations et stimuli - ici, les sensations de chaleur et de lumière d'un jour d'hiver exceptionnel - viennent «jouer» de ses nerfs et les faire vibrer, comme les cordes d'une harpe éolienne.

Dans ce poème, ce n'est pas le vent, mais les sensations empiriques qui jouent de cet instrument. Or, quelques mois auparavant, Coleridge avait écrit l'un de ses plus beaux poèmes, « La harpe éolienne » (1795), qui élabore la figure du cerveau comme instrument de musique à la conjointure des philosophies néoplatonicienne, unitarienne et associationiste, tout en reprenant la signification de sensibilité que lui prêtait la littérature ${ }^{18}$ :

\footnotetext{
16 « Nature's vast ever-acting Energy !»; « The Destiny of Nations », 1796, vers 461. Nos citations des poèmes d'Emerson sont extraites de Collected Works, Kathleen Coburn (éd.), 16 vols., Princeton, Princeton University Press, 1971-2002, vol. 16. Les traductions sont de Danielle Follett.

17 « On Observing a Blossom on the First of February 1796 » :

And the warm wooings of this sunny day

Tremble along my frame, and harmonize

Th'attemper'd brain, that ev'n saddest thoughts

Mix with some sweet sensations, like harsh tunes

Play'd deftly on a soft-ton'd instrument.

${ }^{18}$ Pour les relations entre « The Eolian Harp » et les sources néoplatoniciennes et autres, voir Michael Raiger, « The Intellectual Breeze, the Corporeality of Thought, and "The Eolian Harp" », Coleridge Bulletin : The Journal of the
} 
Et beaucoup de fantaisies fugitives et inutiles,

Traversent mon cerveau indolent et passif,

Aussi sauvages et variées que les bourrasques aléatoires

Qui s'étendent et flottent sur ce luth soumis !

$(\text { vers } 35-44)^{19}$

L'analogie entre la harpe et le cerveau est ici rendue claire : les pensées et les fantaisies qui traversent le cerveau sont comme les «bourrasques aléatoires » qui touchent les cordes de l'instrument. Le cerveau est explicitement "passif», ouvert à toutes les impressions et associations que les stimuli lui apportent. Les pensées ne sont ni appelées ni gardées: elles entrent et repartent aussitôt, comme l'input et l'output d'énergie qui traversent une machine, dans le modèle du cerveau mécanique de Hartley. Le mot «soumis » («subject») souligne la passivité du cerveau : son sens comme adjectif est celui de la sujétion ou de la soumission de la harpe au vent, et non celui du « sujet » lyrique qu'elle pourrait représenter en tant que produisant le chant. Le poème continue, avec sa célèbre question :

Et si toute la nature animée

N'était que des Harpes créées diversement

Qui tremblent et pensent, balayées

Par une seule brise intellectuelle, plastique et vaste,

Simultanément l'âme de chacune, et le Dieu de toutes?

$(\text { vers } 45-49)^{20}$

Friends of Coleridge, $\mathrm{n}^{\mathrm{o}}$ 20, 2002, p. 76-84 ; C. G. Martin, «Coleridge and Cudworth : A Source for "The Eolian Harp" », Notes and Queries, n” 13, 1966, p. 23-25; Herbert Piper, " "The Eolian Harp" Again », Notes and Queries, $\mathrm{n}^{\circ}$ 15, 1968, p. 23-25; M. H. Abrams, "Coledrige's "A Light in Sound": Science, Metascience and Poetic Imagination ", Procedings of the American Philosophical Society, n ${ }^{\circ} 116,1972$, p. 458-476; Richard Berkeley, "Silence and the Pantheistic Sublime in Coleridge's Early Poetry », Coleridge Bulletin, n 24, 2004, p. 59-67; George Dekker, Coleridge and the Literature of Sensibility, op. cit., p. 101-141.

19 "And many idle flitting phantasies, Traverse my indolent and passive brain, As wild and various, as the random gales That swell and flutter on this subject Lute!" "And what if all of animated nature Be but organic Harps diversly fram'd, That tremble into thought, as o'er them sweeps 
Rappelons que les mots «plastique» et « intellectuel»soulignent le rapport avec Cudworth. Or, le fait que cette expression de foi quasi-panthéiste soit donnée sous forme de question («Et si...») est important; c'est à la fois une façon rhétorique de se protéger des accusations d'hétérodoxie, mais aussi une réelle hésitation de la part de Coleridge, très conscient de l'écart entre ces idées et la doctrine chrétienne. Même si Hartley, membre de l'Église d'Angleterre, consacre un grand nombre de pages à la démonstration de la compatibilité de ses théories avec le christianisme, et même si l'unitarisme s'affirme résolument comme chrétien, Coleridge tend à voir une contradiction fondamentale entre le panthéisme et le christianisme.

Sous l'influence de Hartley et de Locke, Coleridge dans ses premiers poèmes identifie l'esprit humain avec la harpe plutôt qu'avec le vent, c'est-à-dire avec la nature passive et réceptrice plutôt qu'avec la force active et nouménale, ce qui représente une rupture par rapport à Cudworth. C'est cette passivité de l'esprit impliquée par le modèle de la harpe éolienne qui conduira Coleridge à le remettre en cause. Vers 1800, Coleridge commence à croire que le panthéisme et l'associationisme vont ensemble et sont à rejeter ensemble comme toute la tradition de l'empirisme britannique. L'automatisme que représente cette figure sera non seulement dénoncé avec force dans les écrits plus tardifs, mais il sera aussi en grande partie la raison même de son refus de tout panthéisme et de son refuge final dans le trinitarisme.

La figure de la harpe éolienne et le néoplatonisme traverseront l'Atlantique pour inspirer à leur tour les transcendantalistes américains. En dépit de l'influence de Coleridge sur Ralph Waldo Emerson, Coleridge ne constitue pour ce dernier ni la source de la figure de la harpe éolienne ni celle de son néoplatonisme. La harpe est connue comme figure littéraire romantique en général ; Emerson l'aurait également trouvée chez Wordsworth, Shelley, Collins, Thomson et d'autres. C'est dans les années 1830 qu'Emerson s’intéresse au néoplatonisme. En 1834 et 1835, il est très influencé par Le Système véritable intellectuel de l'univers de Cudworth. Or, à l'encontre du jeune Coleridge, Emerson rejette Locke et ne s'intéresse pas à la tradition

Plastic and vast, one intellectual breeze,

At once the Soul of each, and God of all ?"

Thomas McFarland écrit à propos de ce passage : «[C'est] un exemple du pur spinozisme néoplatonicien... », Coleridge and the Pantheist Tradition, Oxford, Clarendon Press, 1969, p. 166 ; on peut supposer que McFarland utilise le terme «spinozisme» ici pour désigner le panthéisme en général car, comme il le montre lui-même, Coleridge ne lit pas Spinoza avant 1797 au plus tôt (p. 165). En outre, ce passage n'est pas spinoziste et Spinoza n'était pas néoplatonicien. Spinoza ne préconise pas le même dynamisme que le néoplatonisme ; le panthéisme de Spinoza fut l'une des cibles de Cudworth. 
associationniste de psychologie. Son intérêt pour la musique de la nature reste néoplatonicien. L'émanationnisme néoplatonicien qui fascine Emerson trouve sa grande expression dans l'essai « La Sur-âme » («The Oversoul »), écrit entre 1837 et 1841, et continue d'être important pour lui jusqu'à sa mort. Emerson donne comme épigraphe à l'essai un poème de Henry More. Les courants, flux et forces immanentes qui circulent à travers les strates de l'existence, unifiant le matériel avec le spirituel, sont figurés par le vent, l'eau et le courant de l'électricité. Pour Emerson, cette expression n'est pas seulement métaphorique : le vent est littéralement une force spirituelle immanente. La brise qui balaie les sapins et qui dessine des traces éphémères à la surface de l'étang, est en elle-même la respiration cosmique. À part certaines références à la harpe éolienne dans ses écrits en prose, Emerson lui consacrera un poème, précisément intitulé « La Harpe » (publié en 1876) :

Ce ton mystique ne parle pas de lui-même

Mais seulement des Dieux supérieurs :

Il tremble avec la respiration cosmique, -

Ce qu'il entend, il dit ;

Il obéit humblement à la Cause primale,

Il est la langue des lois du monde.

$(\text { vers } 67-72)^{21}$

Il y a quelques échos de «La harpe éolienne » de Coleridge dans ce poème : ici le mot «tremble», et quelques lignes plus tôt, le poète pose une question semblable à celle de Coleridge : «Et si...» (« And what if... »). Après sa question, Coleridge revient immédiatement dans son poème sur son hétérodoxie et exprime des doutes sur le panthéisme, tandis qu'Emerson décrit les poètes humains qui ne sont pas à la hauteur de la harpe. Tout se passe comme si

\footnotetext{
${ }^{21}$ Nos citations des poèmes d'Emerson sont extraites de Collected Poems and Translations, Harold Bloom et Paul Kane (éd.), New York, Library of America, 1994.

Speaks not of self that mystic tone,

But of the Overgods alone :

It trembles to the cosmic breath, -

As it heareth, so it saith ;

Obeying meek the primal Cause,

It is the tongue of mundane laws.
} 
Emerson façonnait ce poème comme une sorte d'écho au poème de Coleridge, donnant cependant une réponse affirmative et plus détaillée à la question posée par son prédécesseur.

Fait intéressant, Emerson semble ne pas avoir compris que les angoisses suscitées chez Coleridge par l'émanationnisme et la figure de la harpe éolienne, surtout au niveau éthique, l'avaient amené à les rejeter entièrement. Non seulement il passe à côté des doutes profonds de Coleridge quant à la compatibilité de l'immanentisme panthéiste avec la puissance d'agir humaine (agency), mais, plus surprenant encore, il se fait lui-même simultanément le champion d'une sorte de monisme émanationniste et de la confiance en soi (self-reliance). L'angoisse de la contradiction - jugée insoluble par Coleridge vers la fin de sa vie - entre l'Un qui agit dans la multiplicité du monde et le soi qui doit agir avec sa pleine volonté autonome, disparait chez Emerson. Il ne va pas jusqu'à dire que l'homme est comme un automate, mais souvent il accorde la capacité d'agir à une force supérieure à l'homme. Par exemple, dans un poème manuscrit, il écrit :

O quels sont les héros, les prophètes, les hommes,

Sinon des pipes à travers lesquelles souffle la respiration de Pan

Une musique passagère.

$$
(\text { vers } 1-3)^{22}
$$

Pour Emerson, les forces spirituelles ne circulent pas seulement dans la nature, elles entrent aussi dans l'esprit des hommes pour leur apporter à la fois la poésie et les idées en général. Cette approche se distingue légèrement, quoique sur un point important, du néoplatonisme de Plotin, de Cudworth et de More, lesquels ne mettent pas l'accent sur la question de l'esprit individuel humain et de sa capacité d'agir, questions modernes par excellence et romantiques en particulier. Bien que le poème ci-dessus reflète clairement la métaphysique néoplatonicienne de Cudworth, ce dernier n'aurait probablement pas posé cette question de cette manière. Si, pour le néoplatonisme classique, la contradiction entre un esprit humain créateur, analogue à l'Esprit divin, et un esprit humain récepteur des forces divines, analogue donc à la matière, est inhérente et latente, elle ne prend pas une place assez importante

22 O what are heroes, prophets, men,

But pipes through which the breath of Pan doth blow

A momentary music. 
pour donner lieu à un paradoxe contraignant. Pour ces penseurs - si l'on peut généraliser - le modèle des strates concentriques et hiérarchiques suffit à expliquer la place de l'individu humain dans le cosmos, entre 1'Esprit et la matière. La volonté humaine qui fait agir le corps est analogue à la force divine qui fait agir la nature, et aucune contradiction, aucun doute n'apparaît quant à la capacité d'agir de l'homme. Pour les modernes, en revanche, le statut de l'homme dans le cosmos n'est pas aussi aisément décidable. Ses relations avec le divin et la nature sont déstabilisées et les questions philosophiques concernant le sujet humain et les capacités de l'esprit prennent une place centrale. Emerson, à l'instar de Coleridge, ne peut se soustraire à la prise en compte de ces basculements révolutionnaires, " coperniciens »; cherchant à répondre à Locke, qu'il considère comme l'un des responsables de ce tournant moderne, il ne parvient pas à opérer un simple retour au néoplatonisme rejeté par Locke. La centralité de l'esprit humain chez Emerson est donc proprement moderne, romantique et idéaliste, ce par quoi il se distingue du néoplatonisme ancien. Cette situation a pour effet d'exacerber la contradiction, latente dans le néoplatonisme, entre l'esprit humain récepteur et l'esprit humain créateur. Et bien qu'Emerson présente une théorie du soi qui tente de conserver l'équilibre entre les deux activités, entre réception et création, les différentes négociations de ce conflit ne parviendront jamais à le résoudre entièrement puisqu'il est inhérent à la perspective philosophique immanentiste - ce que savait bien Coleridge. D'où certaines tensions célèbres chez Emerson, que les commentateurs ont parfois décrites comme une contradiction entre les principes de liberté et de destin. ${ }^{23}$

L'histoire de la harpe éolienne et de la musique de la nature ne s'arrête pas, c'est clair, avec Emerson. Cette esquisse a nécessairement raccourci une discussion qui mériterait d'être plus longue et, faute de place, elle a laissé de côté d'autres penseurs et poètes qui auraient trouvé leur place ici. Néanmoins, en décrivant les circulations des savoirs entre périodes, pays et domaines, les résumés sont parfois non seulement excusables mais, et espérons que c'est le cas présent, utiles. La circulation de l'idée de la musique de la nature dans des domaines, des périodes et des pays différents n'est pas analogue à la circulation du vent néoplatonicien, éternel, abstrait, nouménal, qui traverse les harpes de la nature. Ce n'est pas la même idée qui passerait telle quelle de Cudworth à Emerson, même si l'un exerce indéniablement son influence sur l'autre ; du XVIIe siècle au XIXe le vent a changé. La dynamique de cette idée s'explique plutôt

\footnotetext{
${ }^{23}$ Voir, entre autres, Stephen Whicher, Freedom and Fate : An Inner Life of Ralph Waldo Emerson, Philadelphia, University of Pennsylvania Press, 1953.
} 
par l'histoire réelle ; elle se transforme sous la contrainte où l'homme se trouve d'avoir à négocier avec la modernité, dans un monde où il a perdu son statut cosmologique privilégié, entre le divin et la nature, dans lequel il doit (comme pour compenser cette perte), se conférer à lui-même le statut éminemment problématique de l'individu capable d'agir. Les mutations de l'idée de la harpe éolienne sont également marquées par l'avènement de l'imaginaire de la machine à l'âge de l'industrialisation, comme l'illustre par exemple l'identification du cerveau à une machine réceptrice d'énergie. La peur coleridgienne de la réduction de l'homme à la machine coïncide avec le mouvement historique du luddisme. Ainsi les idées, y compris l'idée d'origine néoplatonicienne de la musique de la nature, ne constituent-elles pas des abstractions platoniciennes statiques mais sont ancrées dans la contingence et la dynamique de l'histoire. 